assessed the symptoms of upper airway cough syndrome, asthma medication use and associated risk factors. Parents were asked their views of these assessments as an alternative to attending clinic utilising a Likert questionnaire cuing at 1 not at all and at 6 a lot. All children performed pulmonary function tests at the clinic and these were compared to the ACT scores. The RAP was compared to Physician assessment of Asthma and associated co morbidities.

Results One hundred and nine questionnaires were distributed with 102 fully completed. The M: F was 1.8:1. The mean age was 9.1. Asthma severity was mild in 23 (23\%), moderate in 59(59\%) and severe in $18(18 \%)$. The positive predictive value of ACT versus pulmonary function tests was $89 \%$. The RAP identified 19 (18.6\%) children with good asthma control but significant UACS symptoms. Fifty six (55\%) parents would utilise the questionnaire to obviate a clinic visit, if rapid access to the clinic was available.

Conclusion Questionnaire assessment can adequately identify the absence of asthma and UACS symptoms in children and is acceptable to more than half of parents attending an asthma clinic.

\section{REGULATORY T CELLS SUBSETS IN CHILDREN WITH SLE}

doi:10.1136/archdischild-2012-302724.0485

'D Sayed, ${ }^{2} \mathrm{~A}$ Eltayeb, ${ }^{2} \mathrm{~N}$ Afifi, ${ }^{2} \mathrm{MA}$ Ibrahim. ${ }^{\top} \mathrm{Clinical}$ Pathology, South Egypt Cancer Institute Assiut University; ${ }^{2}$ Assiut University, Assiut, Egypt

In the case of SLE, Treg deficiencies have been described in mouse models of SLE. However, there are somehow conflicting data in the literature on whether Treg cells in human SLE are numerically and/ or functionally impaired. We aimed to quantify CD4+CD25+Foxp3+ $\mathrm{T}$ cells in children with SLE and to correlate these findings with their disease activity scores and drug therapy. We enrolled 37 pediatric SLE patients and 20 healthy children. The disease activity was assessed by measuring serum levels of anti-dsDNA antibody and by using scores of SLEDAI. The CD4+CD25+, CD4+CD25 bright and $\mathrm{CD} 4+\mathrm{CD} 25^{\mathrm{dim}}$ cells in patients were significantly increased than controls. There was no significant difference in the FoxP3\% gated on CD4+CD25 bright, CD4+CD25 $5^{\mathrm{dim}}$ and CD4+CD25-cells in patients and controls and between different grades of activity, different lines of treatments and patients outcomes as regards all studied values. There was no significant correlation between any of studied parameters with SLEDAI score except gated lymphocytes which have significant negative correlation. The increase of CD4+ CD25+ T cells in pediatric patients with active SLE may be a result of increased usage of corticosteroids that affect the phenotype of the $\mathrm{T}$ cells without affection on its regulatory suppression function indicated by FoxP3.

\section{PRENATAL ALLERGEN EXPOSURE FACILITATED AIRWAY REMODELING BY AIRBORNE ALLERGEN STIMULI IN POSTNATAL LIFE}

doi:10.1136/archdischild-2012-302724.0486

${ }^{1} \mathrm{JC}$ Chen, ${ }^{2} \mathrm{ML}$ Kuo. 'Department of Pediatric Surgery, Chang Gung Memorial Hospital; ${ }^{2}$ Department of Microbiology and Immunology, College of Medicine, Chang Gung University, Taoyuan, Taiwan R.O.C.

Background Murine asthma models are mainly created through adulthood sensitization, but lack airway remodeling hallmarks.

Aims This study aimed to examine airway remodeling in the prenatally-sensitized murine asthma model.

Methods FVB/N fetuses were exposed to aluminum-free ovalbu$\min (\mathrm{OVA})$ of $50 \mu \mathrm{g}$ on gestational day 14 , and subjected to aerosolized OVA challenge in their postnatal life. Lung sections were examined after hematoxylin-eosin, periodic acid-Schiff, and Masson's trichrome stainings.

Results Following prenatal OVA sensitization, neither the neonate nor the adult showed any evidence of inflammatory cell infiltration and airway remodeling. Postnatal aerosolized OVA stress elicited extensive peribronchial and perivascular eosinophilic inflammation. The allergic airways were plugged by exfoliated epithelia and mucus. We identified two distinct patterns of epithelial desquamation: complete denudation of airway epithelia, exposing fragmented basement membrane; and peeling of columnar epithelia, leaving a single layer of basal cells adherent to basement membrane. There was subepithelial collagenosis in extrapulmonary airways and smooth muscle hyperplasia was evident in terminal airways. Prenatally OVA-primed mice had no mucin-positive goblet cells in intrapulmonary airways as normal mice, but showed goblet cell metaplasia in large intrapulmonary airways even following mechanical saline stress. However, goblet cell metaplasia spread distally towards small terminal airways after aerosolized OVA challenge. Asthma models through adulthood sensitization only exhibited peribronchial or perivascular inflammation and goblet cell metaplasia.

Conclusions Fetal OVA exposure intensified airway responsiveness to airborne OVA stimuli in postnatal life to cause pathognomonic structural alterations in the lung.

\section{THE INFLUENCE OF INHALED CORTICOTHERAPY ON THE GROWTH AND DEVELOPMENT IN ASTHMATIC CHILDREN}

doi:10.1136/archdischild-2012-302724.0487

C Mohor, MB Neamtu, ML Neamtu. Pediatric Clinic Hospital Sibiu, Lucian Blaga University of Sibiu, Sibiu, Romania

Background Inhaled corticotherapy is the main anti-inflammatory controller type therapy in asthmatic children. Impaired growth as a result of long-term corticotherapy remains a disputed issue of topical interest for both endocrinologists, pneumologists and pediatricians

Objective The study evaluates the influence of long term small dose inhaled corticotherapy on growth and somatic development in asthmatic children.

Methods Observational analytical study on 2 samples of subjects. The study group: 100 asthmatic children with small dose inhaled corticotherapy (beclomethasone dipropionate Becotide 200-400 $\mu \mathrm{g} /$ day or fluticasone propionate Flixotide 100-300 $\mu \mathrm{g} /$ day), for 24 months therapy. The control group: 100 healthy children. Both groups were divided in 5 homogeneous age subgroups, between 5-19 years of age. For both groups the relevant anthropometric landmarks for assessing growth were measured in dynamics at every 6 months, in a 2 years follow-up: body height, shank and plant length growth; thorax, skull, hip, shank and arm circumference growth. The statistical SPSS software was utilized and the index $\mathrm{t}$-test was calculated ( $\mathrm{p}>0.84)$.

Results Comparative evaluation of anthropometric indices after 1 year, respectively 2 years of medication in all age subgroups revealed a minimum reducing of the growth rate in the study group without statistical significance.

Conclusions Inhaled corticotherapy in small doses in a long term therapy (2 years) doesn't significantly affect growth and somatic development in asthmatic children.

\section{THE DEFICIT IN IMMUNOGLOBULIN A, AUTOIMMUNITY RISK}

doi:10.1136/archdischild-2012-302724.0488

${ }^{1} \mathrm{M}$ Marc, ${ }^{1} \mathrm{~A}$ Butnar, ${ }^{2} \mathrm{C}$ Corpodean. ${ }^{1}$ Pediatrics; ${ }^{2}$ Neonatology, University of Medicine and Pharmacy 'Iuliu Hatieganu', Cluj-Napoca, Romania

Introduction and objectives The immunodeficiencies (ID), by the subsequent impairing of the immunoregulation, may be at the origin of certain autoimmune diseases (AID).

The deficit in immunoglobulin $\mathrm{A}$ is one of the most frequent ID associated with AID. 